\title{
A Critical Review on Ayurvedic Diagnostic Methods
}

\author{
Review Article
}

\section{Venkat Shivudu $\mathbf{K}^{1^{*}}$}

1. Reader, P.G. Department of Ayurveda Samhitha \& Siddhantha (Basic Principles), TTD'S, S.V. Ayurvedic College, Tirupati.

\begin{abstract}
Ayurveda is a holistic system of life which focuses the way of living along with treatment aspects. The antiquity of Ayurveda goes back to the Vedas and key points were taken from Darsanas too. The pramanas are the means to get the knowledge but Ayurveda implied the pramanas in a unique way as a examination tool (pareeksha) for diagnosis purpose in Atura and understanding of the individual in Swastha. Several types of pareeksha are available in Ayurvedic classics which include Dasavidha pareeksha, Astasthana pareeksha, Shadvidha pareeksha, Trividha pareeksha and the like. Of these, Dasavidha pareeksha is the most significant as it encompasses all other types of examinations. Many of the diagnostic methods mentioned in Ayurveda are not scientifically practised now a day. Some of the above mentioned examinations were given least importance in case taking and has now turned customary many aspects of examination are neglected. In the present study the prime intention was to formularize a standard separate proforma for diagnosis of diseases, based chiefly on the Dasavidha pareeksha to enable the students and practitioners to have a better procedure for diagnosis.
\end{abstract}

Key words: Pareeksha, Dasavidha pareeksha, special proforma

\section{Introduction:}

Disease is a reflection of abnormalities in the normal structural, physiological or psychological aspects of the body. Treatment is aimed in bringing back the normality. Before application of medication a well organised examination should be carried out, for an explicit diagnosis as also for designing proper line of treatment.

In the classical texts of Ayurveda, different methods of examinations adopted

\section{*Corresponding Author:}

\section{Venkat Shivudu K}

Reader,

PG Department of Ayurveda Samhitha \& Siddhantha (Basic Principles), TTDs SV Ayurvedic College, Tirupati. E-mail: kvenkatshivudu@gmail.com by Ayurvedic physicians are described. There are -

1. Dwividha pareeksha

Commentary on Madhava Nidanam by Vijayarakshita.

2. Trividha pareeksha : Charaka

Samhita, Ashtanga Hridaya

3. Chathurvidha pareeksha : Charaka

Samhita

4. Panchavidha pareeksha : Ashtanga

Hridaya

5. Shadvidha pareeksha : Sushruta

Samhita

6. Ashtasthana pareeksha : Yoga

Rathnakara

7. Dasavidha pareeksha : Charaka Samhita

Of these, Dasavidha pareeksha is the most significant as it encompasses all the other types of examinations. In 
Dasavidha pareeksha except the Vikruthi pareeksha, the other nine are applicable to both Swastha and 'Athura', while Vikruthi pareeksha is done solely in a diseased.

The general procedure for case taking now followed in Ayurvedic Institutions is according to the modern pattern. These case sheets if examined would seem to be insufficient in expressing the classical sense. The case sheets usually consist of a part dealing with the history and examinations, as prescribed by modern clinical texts, and another part, small as it is, allotted for Ayurvedic aspects, consisting of Ashtasthana pareeksha, Dasavidha pareeksha, Nidana and Samprapthi, which is given least attention during case taking. Moreover, no specific criteria for assessment are being followed. In the little space provided against each variable like Mootra, Pureesha, Satwa etc, the students or physicians mark as NAD or Pravara, Madhyama, Avara and so on as a routine. For example, in the examination of the urine, one gets satisfied by asking a few mandatory questions like frequency, colour or presence of associated complaints like burning sensation to the patients and marks either NAD or normal.

It was hence found necessary to formulate separate examination sheets for assessment of Prakruthi, Sara, Satwa, etc, by collecting the required points from literature as well as practice. When this was done, each assessment proforma was elaborate. It is quite embarrassing to incorporate a bundle of assessment sheets for studying a person. Each and every examination is not necessary for each and every patient. They are to be logically selected according to the condition of the disease and the patient. In a child, the assessment of Sara, Satwa are meaningless as they are not fully developed. So also, in certain specific diseases specific examination methods related to the disease are sufficient. For example, in case of sudden injuries or simple conditions like Acne vulgaris, all the examinations are not necessary for diagnosis. Hence it has become essential to design a new compendious proforma according to Ayurvedic system by considering various principles of diagnosis, so that a diagnostic procedure better than the existing one, could be adopted by students and practitioners in the field of Ayurveda.

\section{Aims and objectives:}

To design a special proforma incorporating all the pareeksha mentioned in the classics chiefly Dasavidha pareeksha Materials and methods:

\section{STEPS TO DESIGN THE MASTER PROFORMA:}

1. Master proforma is designed keeping in mind the two conditions ie. prakrita (healthy) qualities in part I and vikrita ie. Vikruta or abnormal qualities in part II.

2. Part one consists of Dasavidha preeksha (except vikriti pareeksha) and prakruta qualities of Ashtasthana pareeksha. Part two consists of Vikruthi pareeksha. (dosha, dhatu, mala, vruddhi and kshaya lakshanas and srotas)and abnormal qualities of Ashtasthana pareeksha.

3. The order followed is physical, physiological and psychological attributes explained sequentially.

4. All the individual attributes described under separate pareeksha together with their variables are concised together. For example all variables described under heading "Body" in context of Prakriti pareeksha, Sara pareeksha, Samhana etc. are all considered into one unit.

5. Any repetition met during such process is eliminated.

6. Unpractical points are neglected for example, alpa sukrata, red colour of glans penis etc.

7. For this concised complete master proforma, key chart is designed 
consisting of all the Dasavidha and Ashtasthana pareeksha bhavas. So by the single master proforma, all 10 fold factors and 8 fold factors to be examined are easily elicited.

A proforma is meant to collect the relevant details pertaining to the topic in a particular order. Thus it starts with collection of personal data as Name, Age, Sex, Address, Occupation etc. which helps one to identify and verify the subject. The detailed evaluation of the subject is obtained through various methods. The quite popular methods often met with are through a questionnaire having scores for its different choices, ticking the appropriate answer for the questions, writing the answers in the columns provided, etc. Some make use of a key chart which helps to reduce the evaluation by tallying the correct or appropriate answers. Here, as the details of each point given in the texts are many and some of them similar, answers have been tabulated in a keychart which helps easy assessment. Thus in this format the relevant details are obtained in a very clear manner. For better understanding of this, a detail purview of one of the factors is undertaken. Here, let us observe the factor 'Sara'. The descriptions related to sara as mentioned in the different classical text were collected and each quality was analysed. The excellence of dhatus as sara represent the state of each dhatu in which the sara of rasadhatu is described in twaksara and the rest to the corresponding name itself. The eighth sara sathwasara relates to the qualities of mind and behavioural patterns. By observing and analysing the sara of a patient, one can come to know about his state of health. The defects or deficiency of each quality in a sara depicts the decrease in the essence of that dhatu and he may be prone to the disease. For example, the physical factor, unctuous skin is in seen both in twak sara and rakta sara; mental quality as happiness in twaksara, raktasara, mamsasara, medosara and sukra sara and so on ; And enthusiasm in raktasara, asthisara and satwasara. So as the repetitions are seen in the explanation of sara, it became necessary to arrange each quality and its presence in the saras listed for easy evaluation. Assessment was made much easier by charting the presence of each quality in the eight saras. In this proforma, each sara is described with its qualities and one can observe the presence of a typical quality in many saras as is evident from the latter part of the proforma. All the characters were taken as subheads and the saras were tallied and deduced for convenient evaluation.

The data thus obtained are referred with the key chart and each character is bifurcated at the level of key chart. For example, the happiness quality is seen in mental quality of twaksara, raktasara, mamsasara, medosara and sukrasara while collecting data it is recorded only once and in later state the same "happiness" quality is considered under all such corresponding saras for which happiness is a character such a twaksara, raktasara, mamsasara, medosara and sukrasara. The keychart of the proformas are designed inorder to avoid mistake and repetitions are omitted taking care that nothing is being lost. This helps for an easy assessment as one has to just go through the chart during evaluation. By the same method all different characters under each subheadings of sara proforma are looked for and data collected. In the end whole data thus obtained are bifurcated under corresponding sara headings making use of key chart ; which give idea about the different saras present.

There are total 63 attributes mentioned under sara pareeksha. The key consist of 9 types of sara, under which 63 attributes are distributed.

The name and the different saras and total number of attributes together with the variables are listed below.
i) Twak sara
$-18$
ii) Rakta sara
$-22$ 


$\begin{array}{ll}\text { iii) Mamsa sara } & -29 \\ \text { iv) Medo sara } & -23 \\ \text { v) Asthi sara } & -15 \\ \text { vi) Majja sara } & -19 \\ \text { vii) Sukra sara } & -24 \\ \text { viii) Satwa sara } & -14 \\ \text { ix) Sarva sara } & -18\end{array}$

Here an attempt is made to furnish minimum essential formats by considering the generally described examination in the classical texts.

\section{MASTER PROFORMA WITH KEYCHART, DESCRIPTION AND DISCUSSION.}

\section{CASE-SHEET}

\begin{tabular}{|l|l|}
\hline 1. BUILD & a) Well, \\
& b) Moderate, \\
c) Poor, \\
d) Robust, \\
e) Flabby, \\
f) Lean / weak, \\
g) Tall, \\
h) Short, \\
i) Mighty body, \\
j) Firm, \\
k) Delicate, \\
1) Unshapely, \\
m) Softness of organs, \\
n) Large \& bulky \\
\hline a) Normal, \\
b) Unctuous, \\
c) Soft, \\
d) Smooth, \\
e) Dry / rough, f) Hard, \\
g) Cold, \\
h) Warm, \\
i) Wet, j) Lustrous, \\
k) Fair, \\
1) Slimy, \\
m) Pinkish, \\
n) Dark, \\
o) Cracked, \\
p) Most attractive, \\
q) Wrinkled, \\
r) Prominent veins \& \\
tendons s) Excessive \\
moles \& marks. \\
\hline
\end{tabular}

\begin{tabular}{|c|c|}
\hline $\begin{array}{l}\text { 3. BODY } \\
\text { HAIRS }\end{array}$ & $\begin{array}{l}\text { a) Thin/fine, } \\
\text { b) Delicate, } \\
\text { c) Less numerous d) } \\
\text { Deep rooted, } \\
\text { e) Unctuous, } \\
\text { f) Dry / rough }\end{array}$ \\
\hline 4. HAIRS & $\begin{array}{l}\text { a) Soft \& thin, } \\
\text { b) Lustrous, } \\
\text { c) Sparse \& brown, } \\
\text { d) Thick \& black } \\
\text { e) Cracked, } \\
\text { f) Early baldness g) } \\
\text { Early greying h) Curly } \\
\text { i) Deep-rooted } \\
\text { j) Dry /Rough } \\
\text { k) Brownish-red } \\
\text { (smoky). }\end{array}$ \\
\hline 5. HEAD & $\begin{array}{l}\text { a) Unsteady, } \\
\text { b) Steady, } \\
\text { c) Large }\end{array}$ \\
\hline $\begin{array}{l}6 . \quad \text { FORE- } \\
\text { HEAD }\end{array}$ & $\begin{array}{l}\text { a) Unctuous, } \\
\text { b) Red colour, } \\
\text { c) Prominent, } \\
\text { d) Large /big, } \\
\text { e) Charming \& radiant } \\
\text { appearance, } \\
\text { f) Firm, } \\
\text { g) Beautifully covered } \\
\text { with muscle. }\end{array}$ \\
\hline 7. FACE & $\begin{array}{l}\text { a) Unctuous, } \\
\text { b) Brownish-red } \\
\text { (dark), } \\
\text { c) Red colour, } \\
\text { d) Roughness. } \\
\text { e) Flushed, } \\
\text { f) Wheatish, } \\
\text { g) Pleasant, } \\
\text { h) Worried look i) } \\
\text { Charming \& radiant } \\
\text { appearance. }\end{array}$ \\
\hline 8. EYES & $\begin{array}{l}\text { a) Unctuous, } \\
\text { b) Dry, } \\
\text { c) Round, } \\
\text { d) Wide/long, } \\
\text { e) Small } \\
\text { f) Pleasant, } \\
\text { g) Brownish-red h) } \\
\text { White, }\end{array}$ \\
\hline
\end{tabular}




\begin{tabular}{|c|c|}
\hline & $\begin{array}{l}\text { i) Red, } \\
\text { j) Bluish black k) } \\
\text { Desire cold, } \\
\text { 1) Desire to hot m) } \\
\text { Sticky, } \\
\text { n)Lustreless } \\
\text { o) Prominent } \\
\text { p) Unstable, } \\
\text { q) Stable, } \\
\text { r) Quickly becomes } \\
\text { red, } \\
\text { s) Burning } \\
\text { t) Lids kept open while } \\
\text { sleeping } \\
\text { u) Cheerfulness } \\
\text { v) Charming and } \\
\text { radiant appearance, } \\
\text { w) Well differentiate } \\
\text { white \& black areas. }\end{array}$ \\
\hline $\begin{array}{l}\text { 9. Ears, Nose } \\
\text { \& Lips }\end{array}$ & $\begin{array}{l}\text { a) Unctuous, } \\
\text { b) Red colour, } \\
\text { c) Charming \& radiant } \\
\text { appearance }\end{array}$ \\
\hline $\begin{array}{l}\text { 10. CHEEKS } \\
\text { \& JAWS }\end{array}$ & $\begin{array}{l}\text { a) Firm, } \\
\text { b) Prominent, } \\
\text { c) Beautifully covered } \\
\text { with muscles }\end{array}$ \\
\hline 11.i. TONGUE & $\begin{array}{l}\text { a) Unctuous, } \\
\text { b) Red colour, } \\
\text { c) Dry/rough, } \\
\text { d) White/pale, } \\
\text { e) Yellow, } \\
\text { f) Bluish black g) } \\
\text { Smooth, } \\
\text { h) Slimy, } \\
\text { i) Soft, } \\
\text { j)Toomoist, } \\
\text { k) Hard, } \\
\text { l) Coating, } \\
\text { m) Unsteady, } \\
\text { n) Cold, } \\
\text { o) Warm , } \\
\text { p) Charming \& radiant }\end{array}$ \\
\hline $\begin{array}{l}\text { ii. Sense of } \\
\text { taste }\end{array}$ & $\begin{array}{l}\text { a) Sweet, } \\
\text { b) Bitter, } \\
\text { c) Pungent, } \\
\text { d) Salty, } \\
\text { e) Astringent, } \\
\text { f) Indefinite }\end{array}$ \\
\hline
\end{tabular}

\begin{tabular}{|c|c|}
\hline 12. TEETH & $\begin{array}{l}\text { a) Unctuous, } \\
\text { b) Strong, } \\
\text { c) Round, } \\
\text { d) White, } \\
\text { e) Orderly, } \\
\text { f) Many, } \\
\text { g) Coarse, } \\
\text { h) Small, } \\
\text { i) Firm }\end{array}$ \\
\hline 13. Neck \& & $\begin{array}{l}\text { a) Firm, } \\
\text { b) Prominent } \\
\text { c) Large Shoulder } \\
\text { d) Beautifully covered } \\
\text { with muscle }\end{array}$ \\
\hline 14. CHEST & $\begin{array}{l}\text { a) Firm, } \\
\text { b)Broad \& fleshy } \\
\text { c) Beautifully covered } \\
\text { with muscles }\end{array}$ \\
\hline $\begin{array}{l}15 . \\
\text { ABDOMEN }\end{array}$ & $\begin{array}{l}\text { a) Firm, } \\
\text { b) Plumpy, } \\
\text { c) Beautifully covered } \\
\text { with muscles }\end{array}$ \\
\hline $\begin{array}{l}\text { 16. Palms \& } \\
\text { Soles }\end{array}$ & $\begin{array}{l}\text { a) Unctuous, } \\
\text { b) Dry / rough, } \\
\text { c) Coppery red d) } \\
\text { Cracked } \\
\text { e) Charming \& radiant } \\
\text { appearance }\end{array}$ \\
\hline $\begin{array}{l}\text { 17. COLAR } \\
\text { BONE }\end{array}$ & $\begin{array}{l}\text { a) Prominent, } \\
\text { b) Beautifully covered } \\
\text { with muscle }\end{array}$ \\
\hline 18. NAILS & $\begin{array}{l}\text { a) Unctuous, } \\
\text { b) Rough/dry, } \\
\text { c) Uniform, } \\
\text { d) White, } \\
\text { e) Pink/red } \\
\text { f) Thin \& soft } \\
\text { g) Strong \& shiny } \\
\text { h) Charming \& radiant }\end{array}$ \\
\hline 19. MUSCLES & $\begin{array}{l}\text { a) Bulged calves b) } \\
\text { Flaccid, } \\
\text { c) Firm \& compact, } \\
\text { d) Weakness, } \\
\text { e) Spasticity. }\end{array}$ \\
\hline 20.BONES & $\begin{array}{l}\text { a) Firm \& strong } \\
\text { b) Bony prominence, } \\
\text { c) Well shaped, }\end{array}$ \\
\hline
\end{tabular}




\begin{tabular}{|c|c|c|c|}
\hline & $\begin{array}{l}\text { d) Easily susceptible to } \\
\text { fractures }\end{array}$ & & \multirow{12}{*}{$\begin{array}{l}\text { d) Resonant } \\
\text { e) Dynamic } \\
\text { f) Melodious } \\
\text { g) High pitched } \\
\text { h) Soft-spoken } \\
\text { i) Heavy } \\
\text { j) Deep } \\
\text { k) Talkative } \\
\text { 1) Irrelevant } \\
\text { m) Indistinct } \\
\text { n) Choked } \\
\text { o) Weak } \\
\text { p) Broken } \\
\text { q) Obstructed } \\
\text { r) Change of voice } \\
\text { s) Monopolize. }\end{array}$} \\
\hline \multirow[t]{6}{*}{ 21. JOINTS } & a) Unstable & & \\
\hline & $\begin{array}{l}\text { b) Stable, } \\
\text { c) Firm \& compact }\end{array}$ & & \\
\hline & d) Robust heels & & \\
\hline & e) Soft \& loose f) & & \\
\hline & Beautifully covered & & \\
\hline & $\begin{array}{l}\text { with muscle } \\
\text { g) Prominent }\end{array}$ & & \\
\hline \multirow[t]{11}{*}{ 22. GAIT } & \multirow{11}{*}{$\begin{array}{l}\text { a) Unsteady, } \\
\text { b) Firm steps with } \\
\text { confidence, } \\
\text { c) Quick, } \\
\text { d) Light } \\
\text { e) Well arrived } \\
\text { movements } \\
\text { f) Steady, foot } \\
\text { pressing against } \\
\text { ground } \\
\text { g) Sometimes walk } \\
\text { without confidence } \\
\text { h) Produces sound } \\
\text { while walking }\end{array}$} & & \\
\hline & & & \\
\hline & & & \\
\hline & & & \\
\hline & & & \\
\hline & & \multirow[t]{4}{*}{ 28. SLEEP } & a) Sound, \\
\hline & & & b) Disturbed, \\
\hline & & & c) Excess, \\
\hline & & & $\begin{array}{l}\text { d) Less } \\
\text { g) Day sleep }\end{array}$ \\
\hline & & \multirow{7}{*}{ 29. DREAMS } & \multirow{7}{*}{$\begin{array}{l}\text { a) Roaming on the } \\
\text { mountains, } \\
\text { dwelling on trees and } \\
\text { moving in the sky } \\
\text { b) See forest fire, } \\
\text { lightening and bright } \\
\text { sun rays } \\
\text { c) See water full of } \\
\text { lotus, rows of birds and } \\
\text { clouds }\end{array}$} \\
\hline & & & \\
\hline \multirow{3}{*}{$\begin{array}{l}\text { 23.i) } \\
\text { ACTIVITIES }\end{array}$} & \multirow{3}{*}{$\begin{array}{l}\text { a) Unsteady, } \\
\text { b) Steady, } \\
\text { c) Fast, } \\
\text { d) Slow }\end{array}$} & & \\
\hline & & & \\
\hline & & & \\
\hline \multirow[t]{2}{*}{ ii) Daily works } & \multirow{2}{*}{$\begin{array}{l}\text { a) Less than } 4 \mathrm{hrs} \text {, } \\
\text { b) } 4-10 \mathrm{hrs} \text {, } \\
\text { c) More than } 10 \mathrm{hrs}\end{array}$} & & \\
\hline & & & \\
\hline \multirow{4}{*}{$\begin{array}{l}\text { iii) Feels } \\
\text { tiredness }\end{array}$} & a) Only after hard & \multirow[t]{5}{*}{ 30.HOBBIES } & a) Fond of garlands, \\
\hline & work & & perfumes \& other \\
\hline & b) Do not feel & & decorations \\
\hline & $\begin{array}{l}\text { c) Even after ordinary } \\
\text { work. }\end{array}$ & & \\
\hline \multirow{3}{*}{$\begin{array}{l}24 . \\
\text { EXERCISES }\end{array}$} & \multirow{3}{*}{$\begin{array}{l}\text { a) Daily, } \\
\text { b) Occasionally c) Nil } \\
\text { d) Once in a while } \\
\text { e) Unsteady. }\end{array}$} & & gambling \\
\hline & & \multirow[t]{3}{*}{ 31. HABITS } & a) Nail biting, \\
\hline & & & $\begin{array}{l}\text { b) Speaking lies, } \\
\text { c) Grinding teeth while }\end{array}$ \\
\hline \multirow{2}{*}{$\begin{array}{l}25 . \\
\text { STRENGTH }\end{array}$} & \multirow{2}{*}{$\begin{array}{l}\text { a) Less, } \\
\text { b) Moderate, } \\
\text { c) Maximum }\end{array}$} & & sleeping. \\
\hline & & \multirow[t]{2}{*}{ 32. HABITAT } & $\begin{array}{l}\text { a) Jangala, } \\
\text { b) Anoopa, }\end{array}$ \\
\hline \multirow[t]{3}{*}{ 26. WEALTH } & \multirow{3}{*}{$\begin{array}{l}\text { a) Less, } \\
\text { b) Moderate, } \\
\text { c) More }\end{array}$} & & c) Sadharana. \\
\hline & & \multirow{3}{*}{$\begin{array}{l}\text { 33. Seasons } \\
\text { Preferred }\end{array}$} & a) Autumn, \\
\hline & & & b) Summer, \\
\hline \multirow{2}{*}{$\begin{array}{l}27 . \quad \text { Voice\& } \\
\text { Speech }\end{array}$} & \multirow{2}{*}{$\begin{array}{l}\text { a) Hoarse, } \\
\text { b) Unctuous, } \\
\text { c) Clear, }\end{array}$} & & c) Winter. \\
\hline & & $\begin{array}{l}\text { 34. Aversion } \\
\text { towards }\end{array}$ & $\begin{array}{l}\text { a) Cold } \\
\text { b) Heat. }\end{array}$ \\
\hline
\end{tabular}




\begin{tabular}{|c|c|}
\hline $\begin{array}{l}35 . \quad \text { FOOD } \\
\text { HABITS }\end{array}$ & \\
\hline i. Consumes & $\begin{array}{l}\text { a) Hot food, } \\
\text { b) Cold food, } \\
\text { c) Dry food, } \\
\text { d) Oily food } \\
\text { e) Light food } \\
\text { f) Heavy food } \\
\text { g) Excess } \\
\text { i) Medium } \\
\text { j) Less } \\
\text { k) Ghrita, Ksheera, } \\
\text { Taila \& Mamsa rasa } \\
\text { l) Any 2-3 items m) } \\
\text { Any one of above }\end{array}$ \\
\hline $\begin{array}{l}\text { ii. Rasas } \\
\text { preferred }\end{array}$ & $\begin{array}{l}\text { a) Sweet, } \\
\text { b) Sour, } \\
\text { c) Salty, } \\
\text { d) Spicy, } \\
\text { e) Bitter, } \\
\text { f) Astringent, } \\
\text { g) Shad rasas, } \\
\text { h) 2-5 rasas } \\
\text { i) Only one rasa } \\
\text { j) Not. }\end{array}$ \\
\hline iii. Appetite & $\begin{array}{l}\text { a) Fairly good } \\
\text { b) Aversion to intake, } \\
\text { c) Excessive hunger \& } \\
\text { thirst } \\
\text { d) Irregular } \\
\text { e) Less hunger \& thirst } \\
\text { f) Eats slowly } \\
\text { g) Eats less \& remain } \\
\text { strong h)Eats \& drinks } \\
\text { often }\end{array}$ \\
\hline $\begin{array}{ll}6 . & \text { Sexual } \\
\text { Desire } & \end{array}$ & $\begin{array}{l}\text { a) Weak sex urge, } \\
\text { b) Intense sex urge, } \\
\text { c) Unsteady } \\
\text { d) Attracted by } \\
\text { opposite sex } \\
\text { e) More children's } \\
\text { f) Not having many } \\
\text { children } \\
\text { g) Not liked by } \\
\text { women's. }\end{array}$ \\
\hline $\begin{array}{l}37 . \\
\text { Menstruation }\end{array}$ & $\begin{array}{l}\text { a) Regular } \\
\text { b) Irregular } \\
\text { c) Complaints }\end{array}$ \\
\hline
\end{tabular}

\begin{tabular}{|c|c|}
\hline 38. SWEAT & $\begin{array}{l}\text { a) Unctuous, } \\
\text { b) Less, } \\
\text { c) Excess \& bad smell }\end{array}$ \\
\hline \multicolumn{2}{|l|}{ 39. URINE } \\
\hline i. Colour & $\begin{array}{l}\text { a) Pale, } \\
\text { b) Yellow, } \\
\text { c) White }\end{array}$ \\
\hline ii. Quantity & $\begin{array}{l}\text { a) Excess, } \\
\text { b) Medium, } \\
\text { c) Less }\end{array}$ \\
\hline iii. Frequency & $\begin{array}{l}\text { a) 2-4 times in a day, } \\
\text { b) } 4-6 \text { times in a day, } \\
\text { c) More than } \\
\text { 6times/day } \\
\text { d) Absent in night } \\
\text { e) } 1-2 \text { times in night } \\
\text { f) More than } 2 \text { times } \\
\text { g) }>8 \text { and }<2 \text { in one } \\
\text { day }\end{array}$ \\
\hline iv. Consistency & $\begin{array}{l}\text { a) Unctuous, } \\
\text { b) Slimy, } \\
\text { c) Clear, } \\
\text { d) Normal flow }\end{array}$ \\
\hline v. Odour & $\begin{array}{l}\text { a) Visra gandha, } \\
\text { b) Fruity smell }\end{array}$ \\
\hline $\begin{array}{l}\text { vi. Other } \\
\text { qualities }\end{array}$ & $\begin{array}{l}\text { a) Frothy, } \\
\text { b) Cold, } \\
\text { c) Hot }\end{array}$ \\
\hline \multicolumn{2}{|l|}{ 40. FAECES } \\
\hline i. Colour & $\begin{array}{l}\text { a) Clay/white, } \\
\text { b) Yellow, } \\
\text { c) Green, } \\
\text { d) Brownish red }\end{array}$ \\
\hline ii. Quantity & $\begin{array}{l}\text { a) Excess, } \\
\text { b) Medium, } \\
\text { c) Less }\end{array}$ \\
\hline iii. Frequency & $\begin{array}{l}\text { a) 1-2 times/day } \\
\text { b) More than } \\
\text { 2times/day, } \\
\text { c) Once in } 2 \text { or more } \\
\text { days } \\
\text { d) Expels frequently } \\
\text { e) Expels urine and } \\
\text { faeces in large } \\
\text { quantity. }\end{array}$ \\
\hline iv. Consistency & $\begin{array}{l}\text { a) Semi solid, } \\
\text { b) Unctuous, } \\
\text { c) Dry, } \\
\text { d) Slimy, }\end{array}$ \\
\hline
\end{tabular}




\begin{tabular}{|l|l|}
\hline & e) Soft, \\
& f) Hard, \\
& g) Loose, \\
& h) Sinking down, \\
& i) Floating \\
\hline v.Other & a) Cold, \\
qualities & b) Hot, \\
& c) Constipation, \\
& d) Offensive smell, \\
& e) Frothy \\
\hline 41. & a) Alcohol, \\
ADDICTIONS & b) Smoking, \\
& c) Chewing, \\
& d) Drugs, \\
& e) Others. \\
\hline
\end{tabular}

SATVA:

\begin{tabular}{|c|c|}
\hline $\begin{array}{l}\text { i. Attitude in } \\
\text { general }\end{array}$ & $\begin{array}{l}\text { a) Optimistic } \\
\text { b) Pessimistic } \\
\text { c) Sometimes } \\
\text { optimistic }\end{array}$ \\
\hline $\begin{array}{l}\text { ii. Ability to stick } \\
\text { on to decisions }\end{array}$ & $\begin{array}{l}\text { a) Excellent } \\
\text { b) Moderate } \\
\text { c) Least }\end{array}$ \\
\hline $\begin{array}{l}\text { iii. Attitude of } \\
\text { parents, teachers } \\
\text { and others } \\
\text { regarding your } \\
\text { habits, behavior, } \\
\text { studies, personal } \\
\text { hygiene etc. }\end{array}$ & $\begin{array}{l}\text { a) They appreciate } \\
\text { b) They advise } \\
\text { c) They rebuke. }\end{array}$ \\
\hline $\begin{array}{lr}\text { iv. } & \text { Reverence } \\
\text { towards learned } \\
\text { persons and elders. }\end{array}$ & $\begin{array}{l}\text { a) Always } \\
\text { respectful } \\
\text { b) Not always } \\
\text { c) Least. }\end{array}$ \\
\hline $\begin{array}{lr}\text { v. } & \text { Response } \\
\text { towards } & \text { trivial } \\
\text { issues } & \end{array}$ & $\begin{array}{l}\text { a) Remain calm \& } \\
\text { composed } \\
\text { b) Easily agitated } \\
\& \text { regrets later. } \\
\text { c) Gets agitated } \\
\text { easily, but regains } \\
\text { composure }\end{array}$ \\
\hline $\begin{array}{l}\text { vi. Reaction } \\
\text { towards } \\
\text { frightening } \\
\text { situations }\end{array}$ & $\begin{array}{l}\text { a)Turns pale and } \\
\text { faints } \\
\text { b) Shocked } \\
\text { c) Undeterred. }\end{array}$ \\
\hline $\begin{array}{l}\text { vii. Disposition on } \\
\text { venturing into a } \\
\text { situation }\end{array}$ & $\begin{array}{l}\text { a) Calculative and } \\
\text { analytical }\end{array}$ \\
\hline
\end{tabular}

\begin{tabular}{|l|l|}
\hline & $\begin{array}{l}\text { b) Indifferent at } \\
\text { first but later } \\
\text { thinks over } \\
\text { c) Indifferent and } \\
\text { runs into troubles. }\end{array}$ \\
\hline $\begin{array}{l}\text { viii. Attitude } \\
\text { towards work }\end{array}$ & $\begin{array}{l}\text { a) Highly } \\
\text { enthusiastic } \\
\text { b) Not equally } \\
\text { enthusiastic } \\
\text { c) Least attention, } \\
\text { lack of interest } \\
\text { towards work. }\end{array}$ \\
\hline $\begin{array}{l}\text { ix. Retaining } \\
\text { capacity }\end{array}$ & $\begin{array}{l}\text { a) Retain for a long } \\
\text { time } \\
\text { b) Moderate } \\
\text { c) Only for a short } \\
\text { time }\end{array}$ \\
\hline $\begin{array}{l}\text { x. Considering the } \\
\text { past event / events } \\
\text { which disturbed } \\
\text { him / her most } \\
\text { mentally } \\
\text { physically }\end{array}$ & $\begin{array}{l}\text { A) State during } \\
\text { events - Cool } \\
\text { a) Cisturbed but } \\
\text { b) managed } \\
\text { c) Totally shattered }\end{array}$ \\
\hline $\begin{array}{l}\text { B) Outcome - } \\
\text { a) Positive } \\
\text { b) Neutral } \\
\text { c) Negative }\end{array}$ \\
\hline
\end{tabular}

MENTAL FACULTY

1. Good behaviour

2. Less friendship

3. Intelligence

4. Truthfulness

5. Gratefulness

6. Generosity

7. Adventurous

8. Perseverance

9. Humbleness

10. Greedy

11. Self confidence

12. Forgiveness

13. Happiness

14. Purity

15. More friendship

16. Gentleness

17. Health

18. Excitement

19. Pride 
20. Jealousy

21. Bashfulness

22. Right attitude

23. Stability

24. Straight forwardness

25. Cannot control senses

26. Good fortunes

27. Grasping power

28. Virtuous acts

29. Devotion

30. Enjoyment

31. Enthusiasm

32. Wisdom

33. Endurance

34. Active

35. Skill

36. Power

37. No greediness

38. Successful life

39. Patience

40. Honour

41. Simplicity

42. Irritability

43. Not cultured

44. Not stead fast

45. Stealing nature

46. Quick in understanding \& forgetting

47. Charity

48. Unstable mind

49. Courage

50. Obedience

51. Affection to dependants

52. Dignified

53. Knowledge

54. Cultured

55. Self-controlled

\section{Master Key Chart}

\begin{tabular}{|l|l|l|}
\hline Vata Prakruti & $\begin{array}{l}\text { Pitta } \\
\text { Prakruti }\end{array}$ & $\begin{array}{l}\text { Kapha } \\
\text { Prakruti }\end{array}$ \\
\hline 1. c,f,g,h,l & 1. b,e,k,1 & 1. a,f,d \\
2. e,f,g,m,o,r & 2 & 2 b,c,d,i,j \\
4. e,j,k & c,d,h,m,q, & 4 b,d,h, \\
5. a & s & $5 \mathrm{~b}$ \\
7. b,d & 4 a,c,f,g & 6 d \\
8 b,e,g,n,t & 7 e,h, & 7 g \\
11. a & 8 e,i,k,r & 8 a,d,f,w \\
\hline
\end{tabular}

\begin{tabular}{|c|c|c|}
\hline $\begin{array}{l}\text { 12. g } \\
\text { 16. b,d } \\
\text { 18. b } \\
\text { 19. a } \\
\text { 20. b,d } \\
21 \text { a } \\
\text { 22.a,h } \\
\text { 23.a } \\
\text { 25. a } \\
\text { 26. a } \\
\text { 27. } \\
\text { a,k,l,m,o,p,q } \\
28 . \text { b,f } \\
29 . \text { a } \\
30 . \text { b } \\
31 \text { a,b,c } \\
34 \text { a } \\
35 \quad \text { (i)d,e } \\
\quad \text { (ii) a,b,c } \\
\text { (iii)d,h } \\
36 \text { a,c,f,g }\end{array}$ & $\begin{array}{l}11 \mathrm{~b} \\
12 \mathrm{~h} \\
16 \mathrm{c} \\
19 \mathrm{~b} \\
21 \mathrm{e} \\
22 \mathrm{c} \\
23 \mathrm{c} \\
25 \mathrm{~b} \\
26 \mathrm{~b} \\
27 \mathrm{c}, \mathrm{s} \\
28 \mathrm{f} \\
29 \mathrm{~b} \\
30 \mathrm{a} \\
34 \mathrm{~b} \\
35 \text { (i) b (ii) } \\
\mathrm{a}, \mathrm{e}, \mathrm{f} \quad(\mathrm{iii}) \\
\mathrm{a}, \mathrm{c} \\
36 \mathrm{a}, \mathrm{f}, \mathrm{g} \\
38 \mathrm{c}\end{array}$ & $\begin{array}{l}12 \mathrm{f} \\
14 \mathrm{~b} \\
16 \mathrm{a} \\
18 \mathrm{~g} \\
19 \mathrm{c} \\
21 \mathrm{c} \\
22 \mathrm{f} \\
23 \mathrm{~d} \\
25 . \mathrm{c} \\
26 . \mathrm{c} \\
27 \mathrm{~b}, \mathrm{~d}, \mathrm{e}, \mathrm{h} \\
28 \mathrm{c} \\
29 \mathrm{c} \\
35 \text { (i) c,j } \\
\text { (ii) d,e,f } \\
\text { (iii) e,f,g } \\
36 \mathrm{~b} \\
38 \mathrm{~b}\end{array}$ \\
\hline $\begin{array}{l}\text { Mental faculty: } \\
2,10,22,25 \text {, } \\
42,43,45,46 \text {, } \\
48\end{array}$ & $\begin{array}{l}\text { Mental } \\
\text { faculty: } \\
1,3,7,14,1 \\
9,20,49,51\end{array}$ & $\begin{array}{l}\text { Mental } \\
\text { Faculty: } \\
4,5,6,8,9 \text {, } \\
11,15,21 \text {, } \\
24,50,52 \text {, } \\
54\end{array}$ \\
\hline
\end{tabular}

\section{Sara Pareeksha}

\begin{tabular}{|c|c|c|}
\hline $\begin{array}{l}\text { Twak } \\
\text { sara }\end{array}$ & $\begin{array}{l}2 \mathrm{~b}, \mathrm{c}, \mathrm{d}, \mathrm{j}, 3 \mathrm{a}, \\
\mathrm{b}, \mathrm{c}, \mathrm{d}\end{array}$ & $\begin{array}{l}\text { Mental } \\
\text { Faculty } \\
3,13,17,18, \\
26,30,36,53\end{array}$ \\
\hline $\begin{array}{l}\text { Raktha } \\
\text { sara }\end{array}$ & $\begin{array}{l}1 \mathrm{k}, 2 \mathrm{~h}, 6 \mathrm{a}, \mathrm{b}, \\
\mathrm{e}, 7 \mathrm{a}, \mathrm{c}, \mathrm{i} 8 \mathrm{a}, \\
\mathrm{i}, \mathrm{f}, 9 \mathrm{a}, \mathrm{b}, \mathrm{c}, \\
11 \text { (i) a, b, h, } \\
16 \text { a, c, e, } 18 \mathrm{a}, \\
\mathrm{e}, \mathrm{h}, 25 \mathrm{~b}\end{array}$ & $3,13,31,33$ \\
\hline $\begin{array}{l}\text { Mamsa } \\
\text { sara }\end{array}$ & $\begin{array}{l}6 \mathrm{c}, \mathrm{f}, \mathrm{g} 81, \mathrm{~m}, \\
10 \mathrm{a}, \mathrm{b}, \mathrm{c}, 13 \mathrm{a}, \\
\mathrm{b}, \mathrm{d}, 14 \mathrm{a}, \mathrm{b}, \mathrm{c}, \\
15 \mathrm{a}, \mathrm{b}, \mathrm{c} 17 \mathrm{~b}, \\
21 \mathrm{c}, \mathrm{f}, \mathrm{g}, 25 \mathrm{c}, \\
26 \mathrm{~b}\end{array}$ & $\begin{array}{l}12,13,17,36, \\
37,39,41,53\end{array}$ \\
\hline $\begin{array}{l}\text { Medo } \\
\text { sara }\end{array}$ & $\begin{array}{l}1 \mathrm{k}, 1,2 \mathrm{~b}, \mathrm{p} \\
3 \mathrm{e}, 7 \mathrm{a} 8 \mathrm{a} 9 \mathrm{a} \\
12 \mathrm{a}, 18 \mathrm{a}, 26 \mathrm{~b},\end{array}$ & $\begin{array}{l}13,30,34,36, \\
41\end{array}$ \\
\hline
\end{tabular}




\begin{tabular}{|c|c|c|}
\hline & $\begin{array}{l}27 \mathrm{~b}, \mathrm{f}, \mathrm{h} 38 \mathrm{a}, \\
39 \text { (iv)a, } 40 \\
\text { (iv)b }\end{array}$ & \\
\hline $\begin{array}{l}\text { Asthi } \\
\text { sara }\end{array}$ & $\begin{array}{l}1 \mathrm{a}, 5 \mathrm{c}, 10 \mathrm{a}, \mathrm{b}, \\
12 \mathrm{~b} 13 \mathrm{~b}, \mathrm{c}, \\
17 \mathrm{a}, 18 \mathrm{~g}, 21 \mathrm{~d}, \\
\mathrm{~g}\end{array}$ & $31,33,34$ \\
\hline $\begin{array}{l}\text { Majja } \\
\text { sara }\end{array}$ & $\begin{array}{l}1 \mathrm{~m}, 2 \mathrm{~b}, 3 \mathrm{a}, \mathrm{c}, \\
8 \mathrm{~b}, \mathrm{~d} 21 \mathrm{~g} 25 \mathrm{c}, \\
26 \mathrm{~b}, 27 \mathrm{~b}, \mathrm{~d}, \mathrm{f}\end{array}$ & $38,40,53$ \\
\hline $\begin{array}{l}\text { Sukra } \\
\text { sara }\end{array}$ & $\begin{array}{l}1 \mathrm{~d}, \mathrm{o}, 2 \mathrm{~b}, \mathrm{p}, 8 \mathrm{~h} \\
12 \mathrm{a}, \mathrm{c}, \mathrm{d}, \mathrm{e} \\
18 \mathrm{a}, \mathrm{d} 25 \mathrm{~b}, \\
26 \mathrm{c} 27 \mathrm{~d}, \mathrm{f} \\
36 \mathrm{~d}, \mathrm{~g}\end{array}$ & $\begin{array}{l}13,16,17,36, \\
40\end{array}$ \\
\hline $\begin{array}{l}\text { Satwa } \\
\text { sara }\end{array}$ & $22 b$ & $\begin{array}{l}3,5,14,28, \\
29,31,32,35, \\
39\end{array}$ \\
\hline $\begin{array}{l}\text { Sarwa } \\
\text { sara }\end{array}$ & $\begin{array}{l}1 \mathrm{a}, \mathrm{j}, 22 \mathrm{e}, 25 \mathrm{c} \\
26 \mathrm{c}, 27 \mathrm{~d}, \mathrm{f}, \mathrm{g}, \\
36 \mathrm{~g}\end{array}$ & $\begin{array}{l}11,13,28,30, \\
33,36,40\end{array}$ \\
\hline
\end{tabular}

\begin{tabular}{|l|l|}
\hline Avara & $\begin{array}{l}24 \mathrm{c}, 28 \mathrm{~b}, \mathrm{e}, 32 \mathrm{~b}, \\
33 \mathrm{~b}, 35 \text { (i)m (ii) }\end{array}$ \\
\hline Ahara sakthi & \multicolumn{1}{|c|}{ c, 35 (i) a,d,e,g } \\
Vata & $\begin{array}{l}\text { (ii) a,b,c (iii) d,h } \\
39 \text { (ii) b, 40 (ii)c } \\
\text { (iii) c }\end{array}$ \\
\hline Pitta & $\begin{array}{l}\text { 1b, 35 (i) b,i (ii) } \\
\text { a,e,f (iii) a,c, 39 (ii) } \\
\text { c, 40 (ii) a (iii) d,e }\end{array}$ \\
\hline Kapha & $\begin{array}{l}1 \mathrm{a}, 35 \text { (i) a,c,f,j (ii) } \\
\text { f (iii) d,e,f (iv) e,f,g } \\
39 \text { (ii) a, 40 (ii) b } \\
\text { (iii) a }\end{array}$ \\
\hline Vyayama sakthi & $\begin{array}{l}1 \mathrm{~d}, 23 \text { (ii) c (iii) b, } \\
24 \text { a, , 25 (iii) a }\end{array}$ \\
\hline Pravara & $\begin{array}{l}1 \mathrm{k}, 23 \text { (ii) b (iii) a, } \\
24 \text { d, 35 (iii) d }\end{array}$ \\
\hline Madhyama & $1 \mathrm{f}, 23$ (ii) a (iii) c, \\
& 24 e, 35 (iii) e \\
\hline
\end{tabular}

\section{VIKRITI PAREEKSHA}

\begin{tabular}{|c|c|}
\hline \multicolumn{2}{|l|}{ Samhanana } \\
\hline Pravara & $\begin{array}{l}1 \mathrm{a}, \mathrm{j}, 19 \mathrm{c}, 20 \mathrm{a} 21 \mathrm{c}, \\
25 \mathrm{c}\end{array}$ \\
\hline Madhyama & $1 b, e, 19 b, 21 e$ 25b \\
\hline Avara & $\begin{array}{l}1 \mathrm{c}, \mathrm{f}, \quad 19 \mathrm{~d} 20 \mathrm{~b}, \mathrm{~d} \\
21 \mathrm{a}, 25 \mathrm{a}\end{array}$ \\
\hline \multicolumn{2}{|l|}{ Satwa } \\
\hline Pravara & $\begin{array}{l}22 \mathrm{~b}, 42 \text { (i)a (ii)a } \\
\text { (iii)a (iv)a (v)a } \\
\text { (vi)c (vii)a (viii)a } \\
\text { (ix)a (x)a }\end{array}$ \\
\hline Madhyama & $\begin{array}{l}22 \text { f, } 42 \text { (i)b (ii)b } \\
\text { (iii)b (iv)b (v)c } \\
\text { (vi)b (vii)b (viii)b } \\
\text { (ix)c (x)a }\end{array}$ \\
\hline Avara & $\begin{array}{l}22 \text { a, } 42 \text { (i)c (ii)c } \\
\text { (iii)c (iv)c (v)b } \\
\text { (vi)a (vii)c (viii)c } \\
\text { (ix)b (x)a }\end{array}$ \\
\hline \multicolumn{2}{|l|}{ Satmya } \\
\hline Pravara & $\begin{array}{l}24 \text { a, } 28 \text { a,d, } 32 \text { a, } \\
33 \text { c, } 35 \text { (i)k (ii)g }\end{array}$ \\
\hline Madhyama & $\begin{array}{l}24 \text { b, } 28 \text { f,g, } 32 \mathrm{c}, \\
33 \mathrm{a}, 35 \text { (i)l (ii)h }\end{array}$ \\
\hline
\end{tabular}

\begin{tabular}{|l|l|}
\hline 1. Body & 1) Excessive \\
unctuousness \\
2) Exc. Smoothness \\
3) Exc. roughness \\
4) Hypothermia \\
5) Heaviness \\
6) Congenital \\
deformities \\
7) Pallor \\
8) Emaciation \\
9) Laziness \\
10) Numbness \\
11) Softness \\
12) Flabbyness \\
13) Tremors \\
14) Itching \\
15) Stiffness \\
16) Inflammation \\
17) Swelling \\
18) Varicose veins \\
19) Abscess \\
20) Haemorrhagic \\
disorders \\
21) Fatigue \\
22) Hardness \\
23) Weakness \\
\hline
\end{tabular}




\begin{tabular}{|c|c|}
\hline & $\begin{array}{l}\text { 24) Malaise } \\
\text { 25) Rigidity } \\
\text { 26) Exhaustion } \\
\text { 27) Tumors } \\
\text { 28) Enlarged lymph } \\
\text { glands } \\
\text { 29) Diff. types of pains } \\
\text { 30) Discolouration - } \\
\text { black / yellow / red / } \\
\text { white / blackish-brown } \\
\text { 31) Burning sensation } \\
\text { of hands and feet. }\end{array}$ \\
\hline 2. Skin & $\begin{array}{l}\text { 1) Excessive } \\
\text { unctuousness } \\
\text { 2) Excessive roughness } \\
\text { 3) Lustreless } \\
\text { 4) Cracks } \\
\text { 5) Scaling } \\
\text { 6) Coldness } \\
\text { 7) Warmness } \\
\text { 8) Too moisture } \\
\text { 9) Itching } \\
\text { 10) Paresthesia } \\
\text { 11) Hypereshthesia } \\
\text { 12) Small hairs } \\
\text { 13) Cyanosis } \\
\text { 14) Pigmented patches } \\
\text { 15) Redness } \\
\text { 16) Numbness } \\
\text { 17) Excessive } \\
\text { smoothness } \\
\text { 18) Glossyskin } \\
\text { 19) Pimples } \\
\text { 20) Discolouration } \\
\text { 21) Different skin } \\
\text { diseases } \\
\text { 22) Burning sensation } \\
\text { 23) Premature wrinkling }\end{array}$ \\
\hline 3. Hairs & $\begin{array}{l}\text { 1) Falling of hairs } \\
\text { 2) Greying } \\
\text { 3) Horripilation }\end{array}$ \\
\hline 4. Face & $\begin{array}{l}\text { 1) Redness } \\
\text { 2) Discolouration } \\
\text { 3) Pimples }\end{array}$ \\
\hline 5. Eyes & $\begin{array}{l}\text { 1) Yellowish } \\
\text { 2) Redness } \\
\text { 3) Burning sensation } \\
\text { 4) Itching } \\
\text { 5) Heaviness }\end{array}$ \\
\hline
\end{tabular}

\begin{tabular}{|c|c|}
\hline & $\begin{array}{l}\text { 6) Excessive lacrimation } \\
\text { 7) Pain } \\
\text { 8) Sticky } \\
\text { 9) Conjunctivitis } \\
\text { 10) Ulceration } \\
\text { 11) Pathetic look } \\
\text { 12) Ptosis } \\
\text { 13) Photophobia } \\
\text { 14) Squint } \\
\text { 15) Anxious } \\
\text { 16) Ticks } \\
\text { 17) Loss of vision }\end{array}$ \\
\hline 6. Mouth & $\begin{array}{l}\text { 1) Excess salivation } \\
\text { 2) Dryness } \\
\text { 3) Ulceration } \\
\text { 4) Disgausia } \\
\text { 5) Ageusia } \\
\text { 6) Feeling of sweetness } \\
\text { 7) Saltiness } \\
\text { 8) Bitterness } \\
\text { 9) Astringent } \\
\text { 10) Sourness taste }\end{array}$ \\
\hline 7. Lips & $\begin{array}{l}\text { 1) Dryness } \\
\text { 2) Inflammation } \\
\text { 3) Emaciation } \\
\text { 4) Excessive Growth } \\
\text { 5) Redness } \\
\text { 6) Discoloured }\end{array}$ \\
\hline 8. Tongue & $\begin{array}{l}\text { 1) Coating } \\
\text { 2) Discolouration } \\
\text { 3) Dryness } \\
\text { 4) Cracks } \\
\text { 5) Ulceration } \\
\text { 6) Fissured } \\
\text { 7) Furs } \\
\text { 8) Tremor } \\
\text { 9) Tastelessness } \\
\text { 10) Sweet taste } \\
\text { 11) Saltish } \\
\text { 12) Bitter } \\
\text { 13) Astringent } \\
\text { 14) Sourness. }\end{array}$ \\
\hline 9. Teeth & $\begin{array}{l}\text { 1) Extra teeth } \\
\text { 2) Hypertrophy } \\
\text { 3) Pain } \\
\text { 4) Looseness } \\
\text { 5) Discolouration } \\
\text { 6) Dry/rough } \\
\text { 7) Tartar }\end{array}$ \\
\hline
\end{tabular}




\begin{tabular}{|c|c|}
\hline & $\begin{array}{l}\text { 8) Breaking } \\
\text { 9) Tingling sensation }\end{array}$ \\
\hline 10. Palate & $\begin{array}{l}\text { 1) Dryness } \\
\text { 2) Parched } \\
\text { 3) Excessive coating }\end{array}$ \\
\hline 11. Throat & $\begin{array}{l}\text { 1) Dryness } \\
\text { 2) Tonsillitis } \\
\text { 3) Uvulitis } \\
\text { 4) Epiglosotitis }\end{array}$ \\
\hline 12. Cheek & $\begin{array}{l}\text { 1) Good musculature } \\
\text { 2) Emaciation }\end{array}$ \\
\hline 13. Neck & $\begin{array}{l}\text { 1) Goitre } \\
\text { 2) Cervical adenitis } \\
\text { 3) Broad \& Fleshy }\end{array}$ \\
\hline 14. Chest & $\begin{array}{l}\text { 1) Dyspnoea } \\
\text { 2) Cough } \\
\text { 3) Palpitation } \\
\text { 4) Pain } \\
\text { 5) Empty feeling } \\
\text { 6) Heaviness }\end{array}$ \\
\hline 15. Abdomen & $\begin{array}{l}\text { 1) Distension } \\
\text { 2) Pain } \\
\text { 3) Heaviness } \\
\text { 4) Flabby } \\
\text { 5) Emaciation } \\
\text { 6) Abdominal tumor } \\
\text { 7) Gurgling noise } \\
\text { 8) Discomfort } \\
\text { 9) Drooping and } \\
\text { movements } \\
\text { 10) Regurgitation } \\
\text { 11) Acid Eruptions } \\
\text { 12) Fullness of stomach } \\
\text { 13) Fullness of flanks } \\
\text { 14) Enlargement of } \\
\text { spleen } \\
\text { 15) Awareness of gases } \\
\text { 16) Abdominal lumps } \\
\text { 17) Hypoperistalisis of } \\
\text { intestines } \\
\text { 18) Infection of anus } \\
\text { 19) Haemorroids }\end{array}$ \\
\hline 16. Breast & $\begin{array}{l}\text { 1) Large \& drooping } \\
\text { 2) Emaciation }\end{array}$ \\
\hline 17. Muscles & $\begin{array}{l}\text { 1) Atrophy } \\
\text { 2) Numbuess } \\
\text { 3) Spasticity } \\
\text { 4) Weakness } \\
\text { 5) Toneless }\end{array}$ \\
\hline
\end{tabular}

\begin{tabular}{|c|c|}
\hline & $\begin{array}{l}\text { 6) Granuloma } \\
\text { 7) Myoma } \\
\text { 8) Boils } \\
\text { 9) Flabbiness } \\
\text { 10) Rigidity } \\
\text { 11) Sloughing of flesh } \\
\text { 12) Hardness } \\
\text { 13) Pain }\end{array}$ \\
\hline $\begin{array}{l}\text { 18. Buttocks, } \\
\text { Calves, Arms } \\
\& \\
\text { Genitals }\end{array}$ & $\begin{array}{l}\text { 1) Large \& bulky } \\
\text { 2) Emaciation }\end{array}$ \\
\hline 19. Joints & $\begin{array}{l}\text { 1) Smoothness } \\
\text { 2) Flaccidity } \\
\text { 3) Pain } \\
\text { 4) Prominent } \\
\text { 5) looseness } \\
\text { 6) Deep seated abscess } \\
\text { 7) Feeling of emptiness } \\
\text { 8) Laxity } \\
\text { 9) Crepitus }\end{array}$ \\
\hline 20. Bones & $\begin{array}{l}\text { 1) Pain } \\
\text { 2) Hypertrophy } \\
\text { 3) Deformities } \\
\text { 4) Loss of weight } \\
\text { 5) Feeling of emptiness } \\
\text { 6) Cracking } \\
\text { 7) Extra bones }\end{array}$ \\
\hline 21. Nails & $\begin{array}{l}\text { 1) Dry / rough } \\
\text { 2) Cracking } \\
\text { 3) Discolouration } \\
\text { 4) Brittleness } \\
\text { 5) Morbidity of nails }\end{array}$ \\
\hline $\begin{array}{l}22 . \text { Blood } \\
\text { vessels }\end{array}$ & $\begin{array}{l}\text { 1) Fullness } \\
\text { 2) Collapsing }\end{array}$ \\
\hline 23. Strength & 1) Loss of strength \\
\hline 24. Activities & $\begin{array}{l}\text { 1) Little activities } \\
\text { 2) Limited activity with } \\
\text { great strain }\end{array}$ \\
\hline $\begin{array}{l}\text { 25. Sense } \\
\text { organs }\end{array}$ & 1) Failure of perception \\
\hline 26. Speech & $\begin{array}{l}\text { 1) Irrelevant } \\
\text { 2) Very little }\end{array}$ \\
\hline 27. Appetite & $\begin{array}{l}\text { 1) Loss of appetite } \\
\text { 2) Excessive hunger } \\
\text { 3) Intense thirst } \\
\text { 4) Anorexia } \\
\text { 5) Dyspepsia } \\
\text { 6) Indigestion }\end{array}$ \\
\hline
\end{tabular}




\begin{tabular}{|c|c|}
\hline & $\begin{array}{l}\text { 7) Aversion to food } \\
\text { 8) Loss of digestion } \\
\text { 9) Irregular appetite }\end{array}$ \\
\hline 28. Food & $\begin{array}{l}\text { 1) Aversion to sweet } \\
\text { food } \\
\text { 2) Desire for sour things } \\
\text { 3) Desire for cold foods } \\
\text { habits } \\
\text { 4) Nausea } \\
\text { 5) Vomiting } \\
\text { 6) Desire for hot foods } \\
\text { 7) Desire for meat } \\
\text { adjacent bones } \\
\text { 8) Desires for fatty food } \\
\text { and meat items } \\
\text { 9) Frequent thirst } \\
\text { 10) Belching } \\
\text { 11) Disinclination of } \\
\text { food }\end{array}$ \\
\hline 29. Faeces & $\begin{array}{l}\text { 1) Constipation } \\
\text { 2) Flatulence } \\
\text { 3) Discolouration } \\
\text { 4) Blood Streaked } \\
\text { 5) Mucous } \\
\text { 6) Purulent } \\
\text { 7) Steatorrhea } \\
\text { 8) Undigested matter } \\
\text { 9) altered smell } \\
\text { 10) Parasites } \\
\text { 11) Difficulty in } \\
\text { defecation } \\
\text { 12) Scybalous } \\
\text { 13) Copious } \\
\text { 14) Tenesmus scanty } \\
\text { 15) Foul smell } \\
\text { 16) Retention } \\
\text { 17) Watery } \\
\text { 18) Large quantity } \\
\text { 19) Pain in rectum } \\
\text { 20) Suppression }\end{array}$ \\
\hline 30. Urine & $\begin{array}{l}\text { 1) Increased } \\
\text { 2) Scanty } \\
\text { 3) Dysurea } \\
\text { 4) Mixed with Blood } \\
\text { 5) Discoloured } \\
\text { 6) Redness of urethra } \\
\text { 7) Suppression } \\
\text { 8) Obstruction } \\
\text { 9) Continuous }\end{array}$ \\
\hline
\end{tabular}

\begin{tabular}{|c|c|}
\hline & $\begin{array}{l}\text { 10) Retention } \\
\text { 11) Painful } \\
\text { 12) Turbidity } \\
\text { 13) Itching } \\
\text { 14)Burning } \\
\text { sensation } \\
\text { 15) Altered smell } \\
\text { 16) Altered amount } \\
\text { 17) Infection of genitals } \\
\text { 18) Numbness of } \\
\text { genitals } \\
\text { 19) Pain in the bladder } \\
\text { 20) Feeling of non- } \\
\text { elimination. }\end{array}$ \\
\hline 31. Sweat & $\begin{array}{l}\text { 1) Increased } \\
\text { 2) Itching } \\
\text { 3) Sticky } \\
\text { 4) Absence of sweat } \\
\text { 5) Foul smelling }\end{array}$ \\
\hline 32. Sleep & $\begin{array}{l}\text { 1) Less } \\
\text { 2) Excessive } \\
\text { 3) Insomnia } \\
\text { 4) Disturbed } \\
\text { 5) Irregular } \\
\text { 6) Necessary in day } \\
\text { sleep }\end{array}$ \\
\hline 33.Breathing & $\begin{array}{l}\text { 1) Forceful } \\
\text { 2) Restricted } \\
\text { 3) Aggravated } \\
\text { 4) Frequent } \\
\text { 5) Too short } \\
\text { 6) Deep \& Shallow } \\
\text { 7) Stertorous and painful } \\
\text { 8) Breathlessness } \\
\text { 9) Cough } \\
\text { 10) Groaning } \\
\text { 11) Hiccough }\end{array}$ \\
\hline $\begin{array}{l}\text { 34. Sexual } \\
\text { problems }\end{array}$ & $\begin{array}{l}\text { 1) Intense sex urge } \\
\text { 2) Impotency } \\
\text { 3) Sterility } \\
\text { 4) Little semen } \\
\text { 5) Semen with blood } \\
\text { 6) Ejaculation after a } \\
\text { long time } \\
\text { 7) Pain in the penis \& } \\
\text { scrotum } \\
\text { 8) Seminal calculi } \\
\text { 9) Burning sensation of } \\
\text { pains }\end{array}$ \\
\hline
\end{tabular}




\begin{tabular}{|c|c|}
\hline & $\begin{array}{l}\text { 10) Discomfort in sexual } \\
\text { inter course } \\
\text { 11) Excess quantity } \\
\text { 12) Abortion } \\
\text { 13) Miscarriage } \\
\text { 14) Absence of emission } \\
\text { 15) Inability for } \\
\text { copulation } \\
\text { 16) Inflammation of } \\
\text { genitals } \\
\text { 17) Suppression of } \\
\text { menstruation }\end{array}$ \\
\hline $\begin{array}{l}\text { 35. Mental } \\
\text { qualities }\end{array}$ & $\begin{array}{l}\text { 1) Vertigo } \\
\text { 2) Giddiness } \\
\text { 3) Blackouts } \\
\text { 4) Unconsciousness } \\
\text { 5) Intolerance of sounds } \\
\text { 6) Delirium } \\
\text { 7) Pathetic look } \\
\text { 8) Stupour } \\
\text { 9) Exhaustion } \\
\text { 10) Emptiness of mind } \\
\text { 11) Emotions } \\
\text { 12) Fear } \\
\text { 13) Anger } \\
\text { 14) Confusion } \\
\text { 15) Lack } \\
\text { understanding }\end{array}$ \\
\hline
\end{tabular}

Key Chart: Dosha, Dhatu, Mala Vridhi and Kshaya lakshnas

\begin{tabular}{|c|c|}
\hline $\begin{array}{l}\text { Vata } \\
\text { vridhi }\end{array}$ & $\begin{array}{l}\text { (1) } \\
3,8,10,13,15,21,23,25,29,30 \\
(2) 2,16,20(5) 12(6) 2,9(8) \\
3(15) 1(23) 1(26) 1(28) 6 \\
(29) 1,2(32) 3(35) 2\end{array}$ \\
\hline $\begin{array}{l}\text { Vata } \\
\text { kshaya }\end{array}$ & $\begin{array}{l}\text { (2) } 10,1(6) 1(24) 1(26) 2 \\
(28) 9(35) 9\end{array}$ \\
\hline $\begin{array}{l}\text { Pitta } \\
\text { vriddhi }\end{array}$ & $\begin{array}{l}\text { (1) } 16,30(2) 6,7,8,20,22(5) \\
1,3(6) 8,10(15) 11(28) 7 \\
(30) 14(31) 1(35) 4,6,9\end{array}$ \\
\hline $\begin{array}{l}\text { Pitta } \\
\text { kshaya }\end{array}$ & (2) 3 \\
\hline $\begin{array}{l}\text { Kapha } \\
\text { vridhi }\end{array}$ & $\begin{array}{ccc}(1) & 1,4,5,7,9 & (2) \\
6,15,16,17,20,22,25 & (6)\end{array}$ \\
\hline
\end{tabular}

\begin{tabular}{|c|c|}
\hline & $\begin{array}{l}1,6,7(8) 1(14) 1,2(15) 5, \\
(26) 2(28) 4,5,11 \text { (29) } 5 \text {, } \\
(30) 12(32) 2\end{array}$ \\
\hline $\begin{array}{l}\text { Kapha } \\
\text { kshaya }\end{array}$ & $\begin{array}{l}\text { (1) } 23, \text {, (2) } 2(15) 17,(19) 5 \\
\text { (28) } 7(32) 1 \text { (35) } 2\end{array}$ \\
\hline $\begin{array}{l}\text { Rasa } \\
\text { Vridhi }\end{array}$ & $\begin{array}{l}1-1,4,5,7,9,2-6,16,20,15, \\
5-126-2,9,8-3 \text { 15- } 125-1 \\
26-128-629-1,232-3,35- \\
2\end{array}$ \\
\hline $\begin{array}{l}\text { Rasa } \\
\text { Kshaya }\end{array}$ & $1-3,8,21,26$ \\
\hline $\begin{array}{l}\text { Rakta } \\
\text { Vridhi }\end{array}$ & $\begin{array}{l}2-7,15,19,20,24,305-215- \\
627-130-4\end{array}$ \\
\hline $\begin{array}{l}\text { Rakta } \\
\text { Kshaya }\end{array}$ & $1-322-2282,3$ \\
\hline $\begin{array}{l}\text { Mamsa } \\
\text { Vridhi }\end{array}$ & $\begin{array}{l}1-27,28,12-113-314-4,6 \\
17-14\end{array}$ \\
\hline $\begin{array}{l}\text { Mamsa } \\
\text { Kshaya }\end{array}$ & $12-2,18-2$ 19-2 25-1 \\
\hline $\begin{array}{l}\text { Medo } \\
\text { Vridhi }\end{array}$ & $\begin{array}{l}1-21,24 \text { 15- } 9 \text { 16-1 } 18-1 \\
33-1\end{array}$ \\
\hline $\begin{array}{l}\text { Medo } \\
\text { Kshaya }\end{array}$ & $\begin{array}{l}1-812-2 \quad 15-5,13 \text { 16- } 218- \\
2\end{array}$ \\
\hline $\begin{array}{l}\text { Asthi } \\
\text { Vridhi }\end{array}$ & $9-1,220-2,7$ \\
\hline $\begin{array}{l}\text { Asthi } \\
\text { Kshaya }\end{array}$ & $3-19-3,4$ 19-3 21-5 \\
\hline $\begin{array}{l}\text { Majja } \\
\text { Vridhi }\end{array}$ & $1-55-5$ 19- 4,6 \\
\hline $\begin{array}{l}\text { Majja } \\
\text { Kshaya }\end{array}$ & $20-535-2,3$ \\
\hline $\begin{array}{l}\text { Sukra } \\
\text { Vridhi }\end{array}$ & $34-1,8$ \\
\hline $\begin{array}{l}\text { Sukra } \\
\text { Kshaya }\end{array}$ & $34-5,6,7,9$ \\
\hline
\end{tabular}

\begin{tabular}{|ll|l|}
\hline 1. & Nadi & $\begin{array}{l}\text { Vata }-\mathrm{a}, \mathrm{b}, \mathrm{i} \\
\text { Pitta }-\mathrm{b}, \mathrm{e}, \mathrm{d} \\
\text { Kapha }-\mathrm{c}, \mathrm{f}, \mathrm{g}, \mathrm{h}\end{array}$ \\
\hline 2. Mootra & $\begin{array}{l}\text { Vata }-39 \text { (i) a (ii) b (iii) b,e } \\
\text { (iv) c,f 30 - 8,10,11 } \\
\text { Pitta - 39 (i) d (ii) c (iii) a,d } \\
\text { (iv)c (v) a (vi) b 30 - 4, 14 } \\
\text { Kapha - 39 (i) c (ii) a (iii) } \\
\end{array}$ \\
& $\begin{array}{l}\text { c,f (iv) a,b (v) b (vi) c 30 - } \\
\text { 13 }\end{array}$ \\
\hline 3. Mala & $\begin{array}{l}\text { Vata- 40 (i) d (ii) c (iii) c } \\
\text { (iv) c,f29- 15 }\end{array}$ \\
\hline
\end{tabular}




\begin{tabular}{|c|c|}
\hline & $\begin{array}{l}\text { Pitta - } 40 \text { (i) b,c (ii) a (iii) } \\
\text { b,d (iv)e,g } 29-4,6,17 \\
\text { Kapha - } 40 \text { (i) a (ii) b (iii) a } \\
\text { (iv) a,b,d } 29-5\end{array}$ \\
\hline 4. Jihwa & $\begin{array}{l}\text { Vata (11) (i) - (ii) a,e,f (8) } \\
4,8 \\
\text { Pitta (11) (i) b,e,i (ii) b,c,e } \\
\text { (8) } 5 \\
\text { Kapha-(11) (i) a,d,j (ii) a,d } \\
\text { (8) } 1,7\end{array}$ \\
\hline 5. Sabda & $\begin{array}{l}\text { Vata (27) a,k,l,m,n,o,p,q,o } \\
\text { Pitta (27) c,q } \\
\text { Kapha (27) b,e,f,i }\end{array}$ \\
\hline 6. Sparsa & $\begin{array}{l}\text { Vata- 2e,f,i,g } \\
\text { Pitta }-2 \mathrm{c}, \mathrm{d}, \mathrm{h}, \mathrm{q} \\
\text { Kapha }-\mathrm{b}, \mathrm{c}, \mathrm{d} \\
\end{array}$ \\
\hline 7. Drik & $\begin{array}{l}\text { Vata }(8) \text { b,g,l,m,n } \\
\text { Pitta (8) l,k } \\
\text { Kapha (8) a,h,q }\end{array}$ \\
\hline 8. Akriti & $\begin{array}{l}\text { Vata- (1) c,f,h (2) } 1 \\
\text { Pitta - (1) b (2) m } \\
\text { Kapha - (1) a,d, (2) r }\end{array}$ \\
\hline
\end{tabular}

\begin{tabular}{|l|l|}
\hline Pureeshavaha & $\mathbf{1 5} 1,2,15 \mathbf{2 9} 1,12$, \\
& $13,16,18,19,21$ \\
\hline Swedavaha & $\mathbf{1} 1,2 \mathbf{2} 22 \mathbf{3} 3 \mathbf{3 1}$ \\
& 1,4 \\
\hline Arthavavaha & $\mathbf{3 4} 3,15,17$ \\
\hline
\end{tabular}

\section{CONCLUSION:}

This type of construction was necessary and important because -

1) Set serves as the reference for the investigator.

2) Set is an easy method for understanding all the details related to particular point.

3) Set helps in avoiding the unnecessary repetition of the same point.

4) Set helps in different along one condition from another.

5) Set serves as the best way for assessing the patient within short time with less efforts and with fewer chance of commanding mistaken.

\section{Srotho Pareeksha}

\begin{tabular}{|c|c|}
\hline Pranavaha & $\begin{array}{l}\mathbf{1} 13 . \mathbf{3 3} 1,2,3,4, \\
5,7,8,9,10,11 \mathbf{3 5} \\
2,4\end{array}$ \\
\hline Udakavaha & $\begin{array}{l}\mathbf{1} 17,32 \mathbf{6} 27182, \\
3 \mathbf{1 0} 1 \mathbf{1 1} 1 \mathbf{2 8} 7\end{array}$ \\
\hline Annavaha & $\begin{array}{l}517 \quad \mathbf{1 5} 1,2 \mathbf{2 7} 4,7, \\
8284,5,7,11\end{array}$ \\
\hline Rasavaha & $\begin{array}{l}\mathbf{1} 5,8,11,13,26, \\
32,33,34 \mathbf{2} 23 \mathbf{3} 2 \\
\mathbf{6} 4,5 \mathbf{1 7} 1 \mathbf{2 7} 4,8, \\
\mathbf{2 8} 4,11 \mathbf{3 4} 2\end{array}$ \\
\hline Rakthavaha & 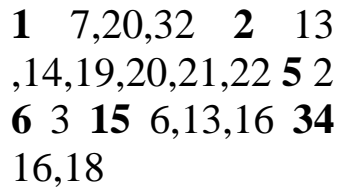 \\
\hline Mamsavaha & 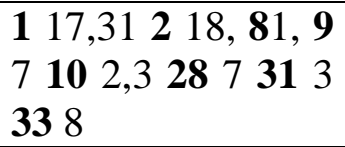 \\
\hline Asthivaha & 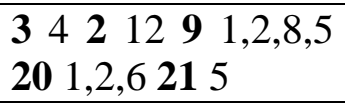 \\
\hline Majjavaha & $\begin{array}{l}\mathbf{1} 5,33 \mathbf{5} 5 \mathbf{1 9} 3,6 \\
\mathbf{2 0} 5 \mathbf{3 5} 2,3 \\
\end{array}$ \\
\hline Sukravaha & $\begin{array}{lccc}\mathbf{1} & 6 & \mathbf{3 4} & 2,3, \\
6,5,1,13,14,15 & \end{array}$ \\
\hline Mutravaha & $301,2,4,7,9,11,18$ \\
\hline
\end{tabular}

\section{References:}

1. Vagbhata 1986 - Ashtanga Hridaya Sarvanga Sundari Commentary, Chowkambha Orientalia, Varanasi.

2. Vridha Vagbhata, (1980) Ashtanga Sangraha, edited by Anant Damodar Atwala, published by Mahesh Anant Atwala, Srimal Atreya Prakasana Nandanandan.

3. Ashtanga Samgraha- Sutra Sthana Samskrita Moola Translated bySri. Panyam Partha Sarathi Sharma (Pariskrita). Vaidya Itikala Sanjeeva Rao. Telugu Academy, Hyderabad 1979. Indukritha Sasilekha Vyakhya.

4. Bhavamisra, (1984)- Bhavaprakasa. Edited with Vidyothini Hindi Commentary Notes and Appendix by Sri Brahmasankara Misra and Sri. Rupalaji Vaisya, Chowkambha Sanskrit Sansthan, Varanasi.

5. Sarngadhara- Sarngadhara Samhitha with Deepika Hindi Commentary by Dr. Brahmananand Tripathi, 
Chowkambha

Granthamala.

Ayurvignan

6. The Students Sanskrit English Dictionary 1993 by Vaman Shivram Apte; Motilal Banarsidas Publishers Private Limited, New Delhi.

7. Prof K.R Srikanta Murthy 1983 Clinical methods in AyurvedaChowkarnbha Orientalia, Varanasi.

8. Ayurveda Mahakosha 1968 Ayurvediya Sabdakosha, by Ayurvedacharya VeniMadhava Sastri \& Ayurveda Narayana Hari Joshi.

9. Vagbhata- Ashtanga Hridaya (1994). English translation by Prof. K.R. Srikanta Murthy, Krishnadas Academy, Varanasi.

10. Rogipareekshavidhi (1982) by Prof. P.V. Sharma, Chowkambha Bharateeya Academy, Varanasi.

11. Bhaishajya Ratnavali- Vidyotini Hindi Vyakhya (1993) by Kaviraja Sri. Ambika Datta Sastri Ayurvedacharya. Chowkambha Sanskrit Sansthan.

12. Agnivesa's Charaka Samhitha (1997) with English translation and critical expostion based on Chakrapanidatta's Ayurveda Deepikas by Dr. Ramkaran Sharma and Vaidya Bhagavan Das. Chowkambha Sanskrit Series, Varanasi.

13. Bhela Samhita (1959). Edited by Sri. Girija Dayalusukla Chowkambha Vidya Bhavan, Varanasi.

14. Introduction to Kayachikitsa by Dwarakanath, Chowkambha Orientalia, Varanasi.

15. Kayachikitsa by Rama Raksha Patak, Chowkambha Publications, Varanasi

16. Kayachikitsa by Shivacharan Das Dhyani.
17. Madhavanidana

Madhukosa Commentary, Chowkambha Orientalia, Varanasi

18. Yogaratnakara- Anonymous with Vidyotini Commentary

19. Sareera kriya Vijnana 2nd Edition, by Dr. M. Ram Sundar Rao.

20. Rogavijnana and Vikriti Vijnana by Subhash Ranade and Paranjape (1984) Publication Division, Ayurveda College, Thiruvananthapuram.

21. Susrutha Samhitha.

Dalhana- Nibanda Samgraha Commentary on Susrutha Samhitha Chowkambha Orientalia, Varanasi,

22. Fundamentals of Ayurvedic Medicine 1980 Vaidya Bhagavan Das, Published by Bansal \& Co New Delhi.

23. Dr. A. Lakshmipathi, A textbook of Ayurveda (Ayurveda siksha).

24. Parameswaran Moosad. T.D. (1959) Amarakosa, Published by Sahithya Pravarthaka Co- operative Society Limited. Kottayam (Reprinted).

25. Raja Radha Kantava (1952) Sabdakalpadruma, Chowkambha Sanskrit Series office, Varanasi,

26. Bhagwan Das and Lalitesh Kasyap (1994), Basic Principles of Ayurveda, Published and printed by Asok.K. Mittal Concept Publishing Company, New Delhi.

27. Basic Principles of Ayurveda - V. B. Athvale

28. Charaka Samhitha, Commentaries of Chakrapani \& Gangadhara.

29. Fundamental Principles of Ayurveda Sri. Dwarakanath.

30. Basic concepts of Ayurveda by Bhagavan Das.

31. Diagnosis and treatment of diseases I \& II by Das \& Kashyap. 